\title{
LA LOGÍSTICA \\ En su Marco Referencial \\ Y CONCEPTUAL
}

\section{RESUMEN}

El propósito de este artículo es realizar una aproximación al marco referencial y conceptual de la logística resaltando los aspectos más sobresalientes. De ahí que inicia con la etimología, el contexto militar y del comercio, haciendo énfasis en sus diferentes conceptualizaciones con las implicaciones que ésta tiene en las organizaciones. Asimismo, se presentan algunas consideraciones de la logística inversa para finalizar con el metalenguaje que ha generado propiciando un repertorio para denominar las nuevas formas comunicativas con un lenguaje especializado en este campo.

\section{CONCEPTUALIZACIONES DE LOGÍSTICA}

Etimológicamente, logística se relaciona con la palabra griega logos (idea). Logística en griego es "Logistike" y se encuentra implícita en la palabra "techne" que quiere decir arte, por lo tanto, se podría interpretar la logística como "La teoría en virtud de la cual la realidad pueda ser calculada lógicamente y predeterminada ${ }^{1 "}$

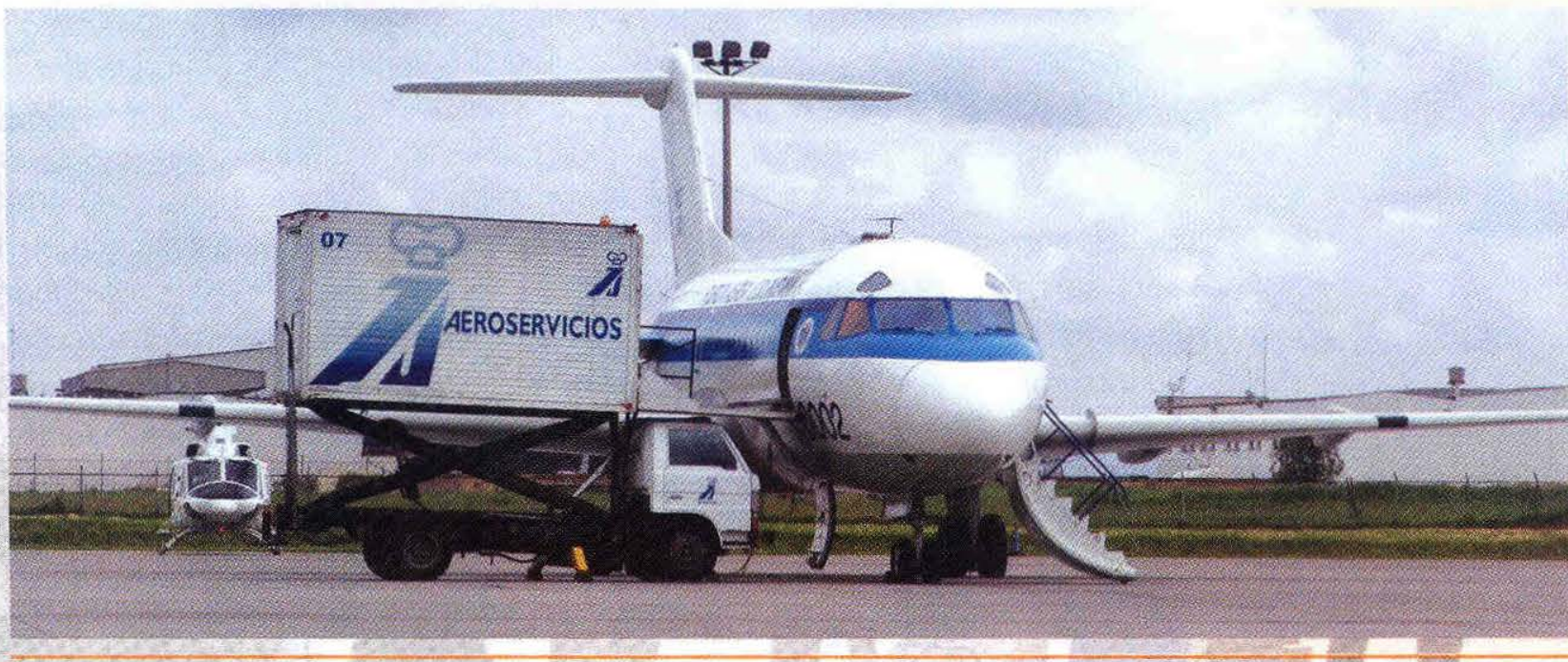

En el diccionario del ejército de los Estados Unidos, el concepto de logística apareció en 1944 y significa planificación de transporte y reabastecimiento. Después fue incluida en los reglamentos que incluía el material, repuestos, suministros e instrucción. Actualmente en el ámbito militar, el concepto de logística incluye planificación de reabastecimiento y transporte; conservación y renovación de la fuerza combativa; normas para el consumo, organización y funcionamiento del aprovisionamiento; repuesto y suministro del material bélico. Se podría afirmar entonces que la logística es el arte de planear y efectuar movimientos militares de transporte, evacuación y refuerzo en todos los sectores. 
En síntesis, la logística sería el nexo de ejecución de las combinaciones de la estrategia y de la táctica. "La logística como parte de la teoría del arte militar" significa el arte de mover las tropas y situarlas, para lo cual se vale de la investigación en lo relativo al enemigo, al territorio para el mejor éxito de las operaciones. Asimismo, constituye el planeamiento operativo de acampar, según las posiciones militares y las exigencias de la estrategia que sustenta y provee al ejército"2. Es así, que la logística es movilizar los ejércitos atendiendo a su seguridad, sostenimiento, dotación de elementos de vida y la condición del combate, realizando planes estratégicos.

De ahí que el Dr. Roberto Bloch, en su documento de aplicaciones militares y civiles, propone la siguiente clasificación de la logística militar como logística estratégica que se ocupa del modo más perfecto de coordinar los movimientos de las tropas, repartiéndolas en varias columnas, en disposición de sostenerse mutuamente por su seguridad, facilitando su descanso, alimentación, efectuando rápidamente las marchas y con la menor fatiga posible para que concurran al combate en las mejores condiciones; mientras que la logística táctica atiende la disposición de los elementos dentro de las columnas, longitud, velocidad de marcha, descansos, colocación de las tropas en los acantonamientos, campamentos, alimentación y seguridad particular. La logística será así el arte del cálculo previo. En la historia de la humanidad el factor logístico ha sido fundamental para el resultado de las guerras.

En términos de comercio, la logística se podría definir como "el proceso de planificar, ejecutar y controlar de forma eficiente el flujo de materias primas, inventarios en curso, productos terminados, servicios e información relacionada desde el punto de origen al de consumo con el fin de satisfacer las necesidades del cliente". ${ }^{3}$

A partir de este enfoque se consideran las actividades logísticas como un número de procesos relacionados con la gestión del flujo de materiales e información del sistema productivo (previsión, gestión de residuos, distribución física, envasado, planificación de la producción y aprovisionamientos).

Actualmente, $y$ ante la necesidad de aumentar la competitividad de las empresas a partir de las herramientas de administración, la logística se ha transformado en una actividad para agregar valor a los negocios del sector empresarial. En 1988, El Council of Logistics Management replanteó la definición anterior y elaboró la siguiente: Gerenciamiento logístico integral que "es la parte de la cadena de abastecimiento integral en la que se planifica, implementa y controla la eficiencia, la efectividad del flujo y almacenamiento de los bienes, servicios y la información relacionada desde el punto de origen hasta el punto de consumo para satisfacer los requerimiento del cliente". ${ }^{4}$ Con este paradigma las empresas deben adaptarse a las nuevas modalidades enmarcadas en la globalización y en la integración del transporte en el ciclo de la producción y el consumo. Debido a esto, las industrias manufactureras introdujeron nuevas técnicas de fabricación y gestión de existencias conocidas con el nombre genérico de logística.

En este sentido, el tema de la logística es esencial, ya que las empresas han diseñado áreas específicas para su tratamiento. Por lo tanto, la logística busca gerenciar estratégicamente la adquisición, el movimiento, el almacenamiento de productos y el control de inventarios; así como el flujo de información asociado a través de los canales de distribución

zPublicar Toolbar Foros Ayuda.

${ }^{3}$ DURAN Heras, Gil Gutiérrez Cas y Chaparro Sánchez Teresa. La logística y el comercio electrónico. Series McGraw-Hill de Managements. Pág. 1.

${ }^{4}$ Monografias.com 
de la organización que se encauzan desde el punto de vista de la rentabilidad presente y futura de la empresa que es maximizada en términos de costos y efectividad. Es decir, que la logística determina y coordina en forma óptima el producto, el cliente oportuno, el lugar correcto y el tiempo justo. Es por esto que la logística no es una actividad funcional sino un modelo, un marco referencial, un mecanismo de planificación para reducir la incertidumbre en el futuro prospectiva.

\section{ACTIVIDADES DE LA LOGÍSTICA}

$\rightarrow$ Transporte.

$\rightarrow$ Gestión de inventarios.

$\rightarrow$ Procesamiento de pedidos

Encaminada a la satisfacción del cliente y a la empresa para la reducción de costos.

\section{FACTORES QUE INTERVIENEN EN LA EVOLUCIÓN DE LA LOGÍSTICA}

$\Rightarrow$ Aumento en las líneas de producción.

$\rightarrow$ Eficiencia en producción.

$\rightarrow$ Cadena de distribución y reducción de los inventarios.

$\rightarrow$ Desarrollo de los sistemas de información.

$\rightarrow$ Estrategias de JIT.

\section{BENEFICIOS DE LA LOGÍSTICA}

$\rightarrow$ Incremento de la competitividad.

$\rightarrow$ Optimización de la gerencia.

$\rightarrow$ Gestión logística con enfoque internacional.

$\rightarrow$ Coordinación óptima de los factores que influyen en la compra: calidad, confiabilidad, precio, empaque, distribución, protección y servicio.

Entonces, se amplia la visión de la logística para convertirla en un modelo y un mecanismo de planificación de las actividades internas y externas de la empresa.

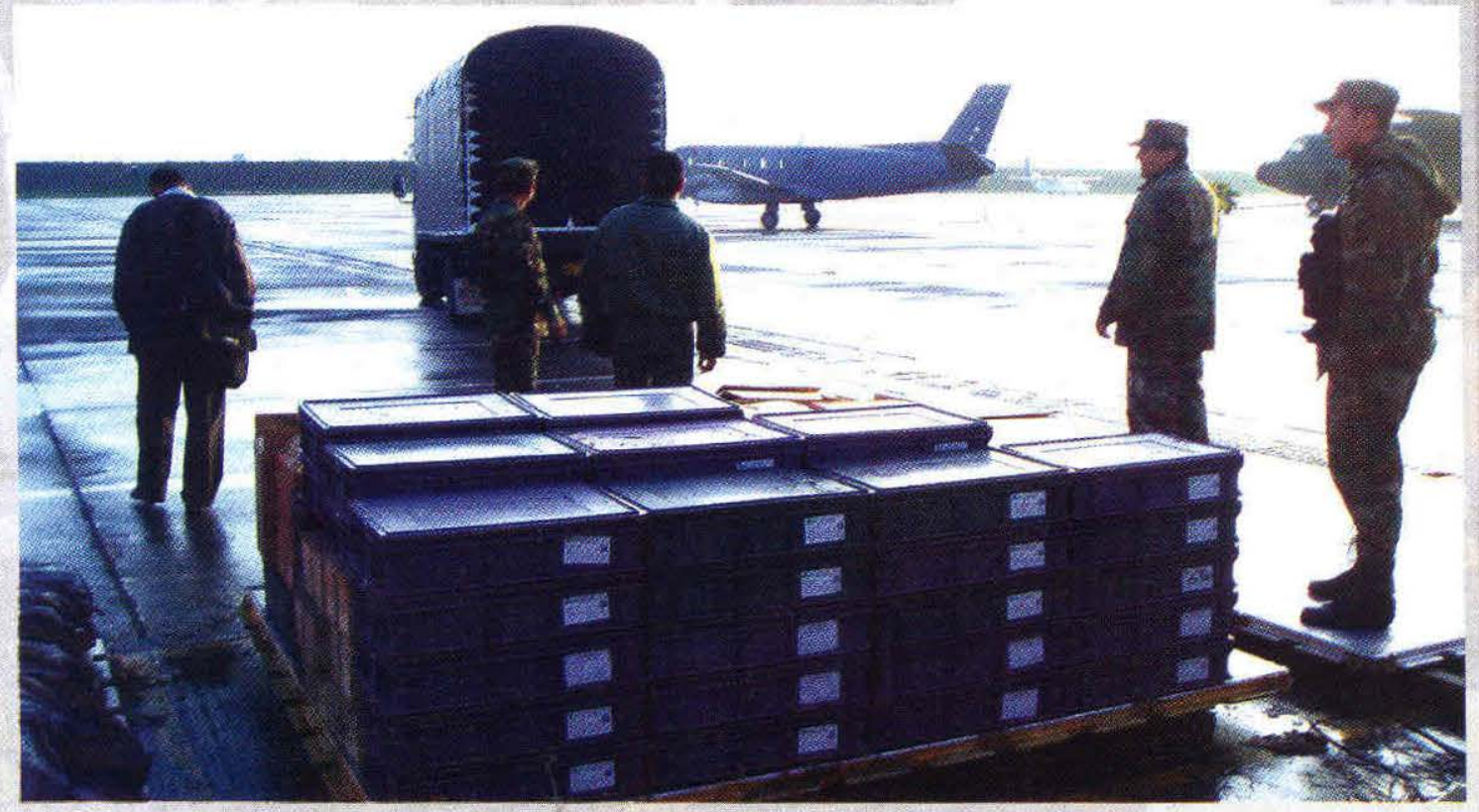




\section{LA LOGÍSTICA DESDE LA PERSPECTIVA DEL CLIENTE IMPLICARÁ:}

$\rightarrow$ Grado de certeza.

$\rightarrow$ Grado de confiabilidad.

$\rightarrow$ Grado de flexibilidad.

$\rightarrow$ Calidad del servicio.

$\rightarrow$ Mejora continua.

$\rightarrow$ Justo a tiempo

\section{LA ADMINISTRACIÓN EN LOGÍSTICA EXIGE LÍDERES EN:}

$\rightarrow$ Una organización logística formal.

$\rightarrow$ Logística a nivel gerencial.

$\rightarrow$ Logística con el concepto del valor agregado.

$\rightarrow$ Orientación al cliente.

$\rightarrow$ Alta flexibilidad en el manejo de situaciones inesperadas.

Las anteriores consideraciones tienen repercusiones en las organizaciones y cada una hace logística en la interrelación natural de los elementos empresariales, internos y externos, de los mercados mundiales, de las economías, los cambios tecnológicos y de la protección del medio ambiente. $Y$ así surge el concepto de logística inversa que es "el proceso de proyectar, implementar y controlar un flujo de materia prima, inventario en proceso, productos terminados e información relacionada desde el punto de consumo hasta el punto de origen de una forma eficiente y económica con el propósito de recuperar su valor o el de la propia devolución". ${ }^{5}$ Entonces, la logística inversa se ha convertido en una herramienta competitiva que establece una política de las devoluciones porque gestiona el retorno de mercancías en la cadena de suministro de forma efectiva y económica, que se encarga de la recuperación y reciclaje de envases. Finalmente, la logística es la promotora de los cambios y transformaciones de los sistemas productivos con proyección en la alta competitividad y aplicación de la teoría CERO en cada una de las actividades empresariales, comerciales, y en el engranaje sistémico de la empresas, abriendo nuevos caminos de táctica y estrategia de negocios, para contextualizarse en la competitividad, la globalización y la superación en el perfeccionamiento de la producción y el consumo.

\section{BIBLIOGRAFIA.}

BLANCHARD S Benjamin. Logistics Engineering and Management. Ed Pearson Prentice Hall sixth edition.

BALLOU H. Ronald. Business Logistics/Supply Chain Management Ed. Pearson Prentice Hall.

BLOCH, Roberto. Aplicaciones Militares y civiles del concepto de logística. Instituto Tecnológico de Bs.As.(ITBA)

DURAN HERAS Alfonso, GUTIERREZ CASAS, Gil y SANCHEZ CHAPARRO Teresa, La logística y el comercio electrónico. Serie MacGraw-Hill de Management www.monografías.com. www.logispilot.com 


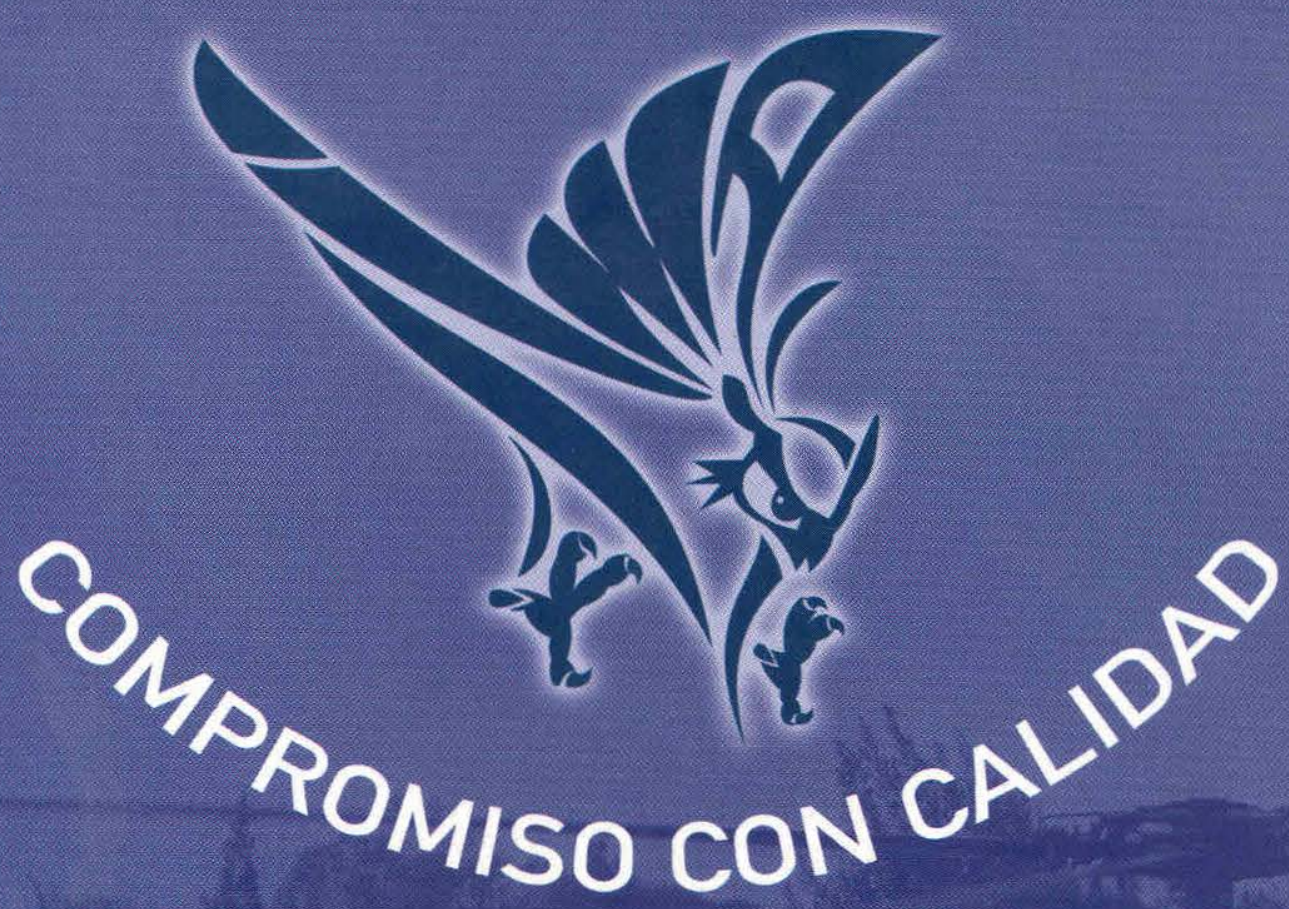

PROGRAMAS:

ESPECIALIZACIONES

Gerencia de la Seguridad Aérea

Logística Aeronáutica

Instituto Militar Aeronáutico

"CAPITÁN JOSÉ EDMUNDO SANDOVAL"

Cra. 11 No. 102 - 50 Tels: 63781276204066 Ext. 4010

Bogotá D.C. - Colombia

PREGRADO

Administración Aeronáutica

Institución Universitaria Resolución 1906 MEN. Agosto de 2002
CURSOS DE EXTENSIÓN

Diplomados en :

Prevención de accidentes

Sicología Aeronáutica

Contratación

Gestión Pública

Planeación Estratégica

Docencia Universitaria

Talento Humano

Cursos de Ascenso

Especialidades Militares

\section{www.ima.edu.co}

